Karlsruhe Reports in Informatics 2019,5 Edited by Karlsruhe Institute of Technology, Faculty of Informatics ISSN 2190-4782

\title{
Using Relational Verification for Program Slicing
}

Bernhard Beckert, Thorsten Bormer, Stephan Gocht, Mihai Herda, Daniel Lentzsch, and Mattias Ulbrich 


\section{Fakultät für Informatik

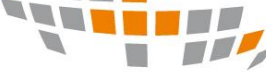 \\ 문}

\section{Please note:}

This Report has been published on the Internet under the following Creative Commons License:

http://creativecommons.org/licenses/by-nc-nd/4.0/de. 


\title{
Using Relational Verification for Program Slicing
}

\author{
Bernhard Beckert, Thorsten Bormer, Stephan Gocht, \\ Mihai Herda, Daniel Lentzsch, and Mattias Ulbrich \\ Karlsruhe Institute of Technology, KTH Royal Institute of Technology
}

\begin{abstract}
Program slicing is the process of removing statements from a program such that defined aspects of its behavior are retained. For producing precise slices, i.e., slices that are minimal in size, the program's semantics must be considered. Existing approaches that go beyond a syntactical analysis and do take the semantics into account are not fully automatic and require auxiliary specifications from the user. In this paper, we adapt relational verification to check whether a slice candidate obtained by removing some instructions from a program is indeed a valid slice. Based on this, we propose a framework for precise and automatic program slicing. As part of this framework, we present three strategies for the generation of slice candidates, and we show how dynamic slicing approaches - that interweave generating and checking slice candidates can be used for this purpose. The framework can easily be extended with other strategies for generating slice candidates. We discuss the strengths and weaknesses of slicing approaches that use our framework.
\end{abstract}

Keywords: program slicing, relational verification

\section{Introduction}

Program slicing, introduced by Weiser [34, is a technique to reduce the size of a program while preserving a certain part of its behavior. Different kinds of slicing approaches have been developed [26]. A static slice preserves the program's behavior for all inputs, while a dynamic slice preserves it only for a particular single input. A backward slice keeps only those parts of the program that influence the value of certain variables at a certain location in the program, while a forward slice keeps those program parts whose behavior is influenced by the variables' values. The form of slicing introduced by Weiser is now known as static backward slicing and is the form of slicing which is pursued in this paper. Slicing techniques can be used to optimize the results of compilers. Slicing is also a powerful tool for challenges in software engineering such as code comprehension, debugging, refactoring, and fault localization [6], as well as in information-flow security [16].

All applications of slicing benefit from small and precise slices. Most existing slicing approaches, however, are only syntactical, i.e., they do not take the semantics of the various program operations into account. On the other hand, the existing approaches that do take the semantics into account are not fully 


$$
\begin{aligned}
& \frac{P[p c]=s k i p}{(s, p c) \rightsquigarrow(s, p c+1)} \\
& \frac{p c>\operatorname{len}(P)}{(s, p c) \rightsquigarrow(e n d, p c)} \\
& \frac{P[p c]=j n z v \text { target } \quad s(v)=0}{(s, p c) \rightsquigarrow(s, p c+1)} \\
& \frac{P[p c]=j n z v \text { target } \quad s(v) \neq 0}{(s, p c) \rightsquigarrow(s, \text { target })} \\
& \frac{P[p c]=\text { halt }}{(s, p c) \rightsquigarrow(e n d, p c)} \\
& \overline{(e n d, p c) \rightsquigarrow(e n d, p c)} \\
& \frac{P[p c]=\text { assign } v \exp \quad x=s(\exp )}{(s, p c) \rightsquigarrow(s[v \backslash x], p c+1)}
\end{aligned}
$$

Fig. 3: The semantics of our programming language for a fixed program $P$

We will now define the semantics of our IR language. Let Var be the set of program variables, $S$ the set of states, where a state is a function $s: \operatorname{Var} \rightarrow \mathbb{N}$, and $p c \in \mathbb{N}$ the program counter. An instruction $I$ is an atomic operation that can be executed by the machine. Let $\mathcal{I}$ be the set of all four instructions provided by our IR language. When an instruction is executed, the system changes its state and program counter as determined by the transition function $\rho: S \times \mathbb{N} \times \mathcal{I} \rightarrow$ $S \times \mathbb{N}$. A program $P$ is a finite sequence of instructions: $\left\langle I_{0}, I_{1}, \ldots I_{n}\right\rangle$. We denote a location $i$ of program $P$ as $P[i]$ with $P[i]=I_{i}$ for any $i \in\{0,1, \ldots n\}$ with $0 \leq i \leq \operatorname{len}(P)-1$, where $\operatorname{len}(P)$ is the length of the program.

The semantics of the four instructions in our IR language is shown in Fig. 3. The instruction skip increments the program counter and has no other effects. To obtain a slice candidate, instructions in the original program are replaced with skip. To model the termination of programs we introduce a special state, end, such that once the system reaches this state, it will remain in this state forever. The instruction halt is used to bring the system to the end state. The assignment instruction, assign, takes a variable $v$ and an integer expression exp as arguments. After the execution of this instruction, the value of the variable $v$ in the new state is updated with the result $x$ of the expression exp and the program counter is incremented. To obtain precise slices, we restrict exp to only one operator. The conditional jump instruction, jnz, allows the register machine to support branching and looping. The instruction gets a variable $v$ and an integer expression target as arguments. If the variable $v$ evaluates to zero in the state in which $j n z$ is executed, then the program counter is incremented, otherwise the program counter is set to the value of target. We will now define program traces:

Definition 1 (Program trace). A trace $T$ of a program $P$ is a possibly infinite sequence of state and program counter pairs $\left\langle\left(s_{0}, p c_{0}\right),\left(s_{1}, p c_{1}\right), \ldots\right\rangle$ such that:

1. $p c_{0}=0$

2. For each trace index $i$ but the last, $\left(s_{i}, p c_{i}\right) \rightsquigarrow\left(s_{i+1}, p c_{i+1}\right)$ 
We use $T^{s}[i]$ and $T^{p c}[i]$ to denote respectively the $i$ th state and the $i$ th program counter of a trace. Also we use $\operatorname{len}(T) \in \mathbb{N} \cup\{\omega\}$ to denote the length of trace $\mathcal{T}$; note that it can be infinite. We define $F_{T}^{l}$ to be the sequence comprised of those states $T^{s}[i]$ for which $T^{p c}[i]=l$, in the same order as they appear in $T^{s}$. We define the notions of a slicing criterion, slice candidate and valid slice:

Definition 2 (Slicing Criterion). A slicing criterion $C$ for a program $P$ is a pair $\left(i_{C}, \operatorname{Var}_{C}\right)$ where $i_{C}$ is a location in $P$ and $\operatorname{Var}_{C} \subseteq \operatorname{Var}$.

Definition 3 (Slice Candidate). A slice candidate for a program $P_{o}$ is a program $P_{L}$ that is constructed by replacing the instructions at some locations in $P_{o}$ with the skip instruction. That is, given a set $L$ of locations of program $P_{o}$ :

$$
P_{L}[i]= \begin{cases}\text { skip, } & i \in L \\ P_{o}[i], & i \notin L\end{cases}
$$

Definition 4 (Valid Slice). Given a slicing criterion $\left(i_{C}, \operatorname{Var}_{C}\right)$, a slice candidate $P_{s}$ for a program $P_{o}$ is a valid slice for $P_{o}$ if, for any two traces $T_{s}$ of $P_{s}$ and $T_{o}$ of $P_{o}$ with $T_{s}[0]=T_{o}[0]$, the following holds:

1. $\operatorname{len}\left(F_{T_{o}}^{i_{C}}\right)=\operatorname{len}\left(F_{T_{s}}^{i_{C}}\right)$,

2. $F_{T_{o}}^{i_{C}}[i](v)=F_{T_{s}}^{i_{C}}[i](v)$ for every $v \in \operatorname{Var}_{C}$ and every $i$ with $0 \leq i<\operatorname{len}\left(F_{T_{o}}^{i_{C}}\right)$.

The first requirement ensures that the criterion location is reached in both the original program and the slice candidate the same number of times. The second requirement ensures that the criterion variables have the same values every time the criterion location is reached in the original program and in the slice candidate.

Weiser 34] deals with the feature-richness of programming languages by working on flow graphs, and slices are constructed by removing nodes from the flow-graph. In his approach, however, only nodes with a single successor can be removed while we can remove conditional jumps. Definition 4 is similar to the concept of observation windows in 34; however, we do not require the original program to terminate. Thus, Definition 4 is more general than that of Weiser.

\section{Relational Verification of Slice Candidates}

Relational verification is an approach for establishing a formal proof that if a relational precondition holds on two respective pre-states of two programs $P$ and $Q$ then the respective post-states of $P$ and $Q$ will fulfill a relational postcondition. For two complex programs that yet are similar to each other, much less effort is required to prove their equivalence than to prove that they both satisfy a complex functional specification. The effort for proving equivalence mainly depends on the difference between the programs and not on their overall size and complexity. This is particularly beneficial for the verification of slice candidates, because the candidates are obtained by replacing program instructions with skip and thus have a structure similar to the original program. 
We formally define the property that is checked by a relational verifier. To that end, we call a predicate $\pi$ a transition predicate for a program $P$ if for any two states, $s$ and $s^{\prime}, \pi\left(s, s^{\prime}\right)$ holds if and only if program $P$ when started in state $s$ terminates in state $s^{\prime}$. Thus, for two programs, $P$ and $Q$, a relational verifier checks the validity of the following proof obligation:

$$
\operatorname{Pre}\left(s_{P}, s_{Q}\right) \wedge \pi\left(s_{P}, s_{P}^{\prime}\right) \wedge \rho\left(s_{Q}, s_{Q}^{\prime}\right) \rightarrow \operatorname{Post}\left(s_{P}^{\prime}, s_{Q}^{\prime}\right),
$$

where $\pi$ and $\rho$ are transition predicates for $P$ and $Q$, respectively, and Pre and Post are respectively the relational precondition and postcondition.

However, a relational verifier that only checks this property is of limited use for checking slice candidates. For the case in which the location of the slicing criterion refers to the post-state (in Fig. 2a that corresponds to location 12 that contains the halt instruction), relational verification can be used to check whether a slice candidate is a valid slice. For a slice candidate $Q$ obtained from a program $P$, this is done by setting Pre to require equal pre-states $s_{P}$ and $s_{Q}$ and Post to require the criterion variables to evaluate to the same values in the post-states $s_{P}^{\prime}$ and $s_{Q}^{\prime}$. However, a successful proof shows the validity of the slice candidates only for inputs for which both $P$ and $Q$ terminate, as the transition predicates may be false for certain pre-states. In the rest of this section we show how a relational verifier can be adapted to support slicing on locations other than the end of the program and how to use relational verification to also show that the program and candidate run in lockstep (i.e. the two executions run through corresponding states), ensuring thus mutual termination.

Our slicing framework is based on the LLRÊVE 12 21 relational verifier, which works on programs written in LLVM IR. It analyzes the control flow graphs (CFGs) of the programs and reduces the validity of the relational specification to the satisfiability of a set $M$ of Horn-constraints over uninterpreted predicates. The satisfiability of the Horn-constraints in $M$ can be checked with state of the art SMT solvers such as Z3 [27] and ElDARICA [29.

If the analyzed programs contain loops, their CFGs contain cycles, which constitute a challenge for verification because the number of iterations is unknown. LLRÊVE handles cycles by using so called synchronization points, at which the program state is abstracted by means of predicates. The paths between synchronization points are cycle free and can be handled easily. Synchronization points

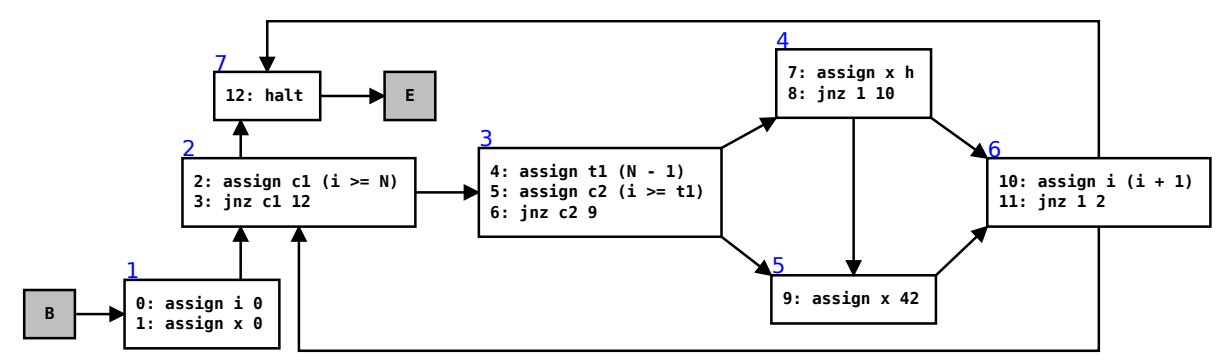

Fig. 4: The CFG for the program in Fig. 2a 
are defined by labeling basic blocks of the CFG with unique numbers. The entry and the exit of a function are considered special synchronization points $B$ and, respectively, $E$. Additionally, the user can also define synchronization points at any location of the analyzed programs. The user must ensure that there is a synchronization point for each basic block of the CFG of the two programs, and has to match them appropriately. In general, it is difficult to find matching synchronization points for two programs; however, in the case of program slicing this can be done automatically by keeping the CFG of the original program. Figure 4 shows the CFG for the program in Fig. 2a and each basic block is labeled with the number of a synchronization point. In the CFG of the slice in Fig. 2b, the assign instruction in block 4 is replaced with skip, the synchronization points remain the same, and matching them is trivial. If a conditional jump is replaced with skip, we only remove the edge to the block containing the jump target, thus keeping the same synchronization points for the slice candidate.

Given one synchronization point per basic block, the CFG can be viewed as a set of linear paths $\langle n, \pi, m\rangle$, where $n$ and $m$ denote the starting and end synchronization points of the path, and $\pi\left(s, s^{\prime}\right)$ is the transition predicate between the two synchronization points, with $s$ and $s^{\prime}$ being the states before and, respectively, after the transition. Because the linear paths consists of assignments only, the transition predicates can be easily computed. For two programs with a similar structure, it is expected that there exist coupling predicates that describe the relation between the program states at two corresponding synchronization points. For two programs $P$ and $Q$ we introduce an uninterpreted coupling predicate $C_{n}\left(s_{p}, s_{q}\right)$ for each synchronization point $n$, as shown in Fig. 5. The relational precondition Pre and postcondition Post are the coupling predicates for the special synchronization points $B$ and $E$, respectively. The set $M$ consists of Horn-constraints over these coupling predicates. For two linear paths between synchronization points $n$ and $m$ in programs $P$ and $Q$ characterized by the two transition predicates $\pi$ and $\rho$, respectively, this constraint is added to $M$ :

$$
C_{n}\left(s_{p}, s_{q}\right) \wedge \pi\left(s_{p}, s_{p}^{\prime}\right) \wedge \rho\left(s_{q}, s_{q}^{\prime}\right) \rightarrow C_{m}\left(s_{p}^{\prime}, s_{q}^{\prime}\right)
$$

To ensure that there is no divergence from lockstep, for every two paths $\langle n, \pi, m\rangle$ and $\langle n, \rho, k\rangle$ in programs $P$ and $Q$, respectively, with $m \neq k, m \neq n, n \neq k$ the following constraint is added to $M$ :

$$
C_{n}\left(s_{p}, s_{q}\right) \wedge \pi\left(s_{p}, s_{p}^{\prime}\right) \wedge \rho\left(s_{q}, s_{q}^{\prime}\right) \rightarrow \text { false }
$$

Theorem 1. Let $P$ and $Q$ be programs specified with the relational precondition Pre and postcondition Post, for which matching synchronization points have been found. Let $M$ be the set of constraints generated according to 1 and 2 . If $M$ is satisfiable, then for every pair of pre-states satisfying Pre:

1. The synchronization points are reached in the same order in $P$ and $Q$,

2. If $P$ terminates, then so does $Q$ and Post holds for the two post-states.

Proof. For distinct synchronization points $n, m, k$ if constraint 2 has a model, then (case 1) $\pi$ or $\rho$ is false, meaning that the execution of $P$ or $Q$ cannot 


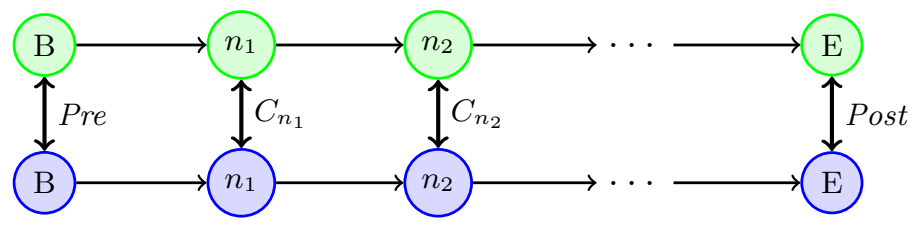

Fig. 5: Illustration of coupled control flow of two fully synchronized programs

reach respectively $m$ or $k$ from $n$, or (case 2) $C_{n}$ is false meaning that $n$ is not reachable in $P$ or $Q$, or per (chaining of) constraint 1 the pre-states do not satisfy the precondition. Thus, $P$ and $Q$ reach the synchronization points (including $E$, thus implying mutual termination) in the same order. For two synchronization points $n, m$ if constraint 1 has a model, then (case 1) $m$ cannot be reached from $n$ in $P$ or $Q$, or (case 2) $C_{n}$ is false and $n$ is not reachable or the pre-states do not satisfy the precondition, or (case 3) starting in $n$ with $C_{n}$ holding, both programs reach $m$ and $C_{m}$ holds there. The constraints generated according to 1 are thus interpolants that show the validity of the relational specification.

To check the validity of a slice candidate for the cases in which the criterion location is in the middle of the program, we adapt the constraints generated by the relational specification. The relational precondition Pre still requires equal pre-states, while the relational postcondition Post is set to true. We ensure a synchronization point $n_{C}$ exists in the program and slice candidate at the location of the criterion instruction. For example $2 \mathrm{a} n_{C}$ is the synchronization point 5 in Fig. 4. If the criterion location is part of a basic block with more than one instruction, we split that basic block up such that we obtain a block containing only the criterion location. For a program $P$ with a slice candidate $Q$ and a given slicing criterion $\left(i_{C}, V_{C}\right)$ with a synchronization point $n_{C}$ we add the following constraint:

$$
C_{n_{C}}\left(s_{P}, s_{Q}\right) \rightarrow \forall x \in V_{C} s_{P}(x)=s_{Q}(x)
$$

Theorem 2. Let $P$ be a program and $Q$ a slice candidate specified with the relational precondition Pre requires equal pre-states and postcondition Post is true. Let $M$ be the set of constraints generated according to 1, 2 and 3. If $M$ is satisfiable, then for every pair of pre-states that fulfill Pre:

1. The criterion location is reached equally often in $P$ and $Q$,

2. Every $i$-th time the criterion instruction is reached, the criterion variables are equal in $P$ and $Q$,

3. If $P$ terminates, then so does $Q$.

Proof. From Theorem 1 results that $P$ and $Q$ run in lockstep with respect to the synchronization points. The instruction at the criterion location has its own synchronization point. As a consequence of this, the criterion instruction is executed in both $P$ and $Q$ the same number of times and the candidate terminates iff the original program terminates. Due to Constraint 3 , the coupling predicate 
corresponding to the criterion locations ensures that each time the criterion location is reached, the criterion variables have the same values.

Thus, for a program $P$ with a slice candidate $Q$ and a slicing criterion $\left(i_{C}, V_{C}\right)$, if the set $M$ containing the constraints 1,2 and 3 for every synchronization point is satisfiable, then $Q$ is a valid slice according to Definition 4 . Moreover, if the set $M$ is unsatisfiable, then the SMT solver returns an unsatisfiability proof that contains a counterexample with two concrete inputs for which the slice property is violated - provided the SMT solver does not time out.

\section{A Framework for Automatic Slicing}

Being able to use relational verification to check whether a slice candidate is valid, we construct a framework for automatic program slicing. The framework, shown in Fig. 6, consists of two components which interact with each other. The first component, the candidate generation engine, generates the slice candidates and sends them to the second component, the relational verifier (in this case LLRÊVE). The relational verifier transmits one of three possible answers to the candidate generation engine: (1) the candidate is a valid slice, (2) the candidate is not valid along with an input that leads to a violation of the slice property (Definition 4), or (3) a timeout. The candidate generation engine can use the answer to adapt its candidate generation strategy.

An advantage of the framework is that the candidate generation engine does not need to care about the correctness of the slice candidates it generates- as this is taken care of by the relational verifier. The framework can easily be extended with candidate generation strategies other than those that we present in this paper. Thus, it provides a platform for relational verification based slicing for the software slicing community.

We distinguish between two types of candidate generation strategies. On the one hand there are strategies that generate candidates by replacing program instructions by skip according to some heuristics not using any information from the relational verifier other than the existence of a counterexample. Examples for such properties are described in Section 4.1. On the other hand there are strategies that also consider the values from the counterexample when generating

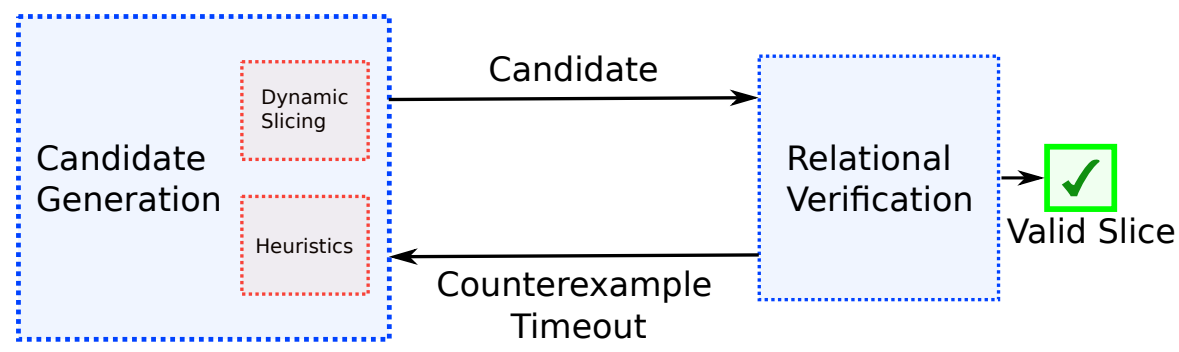

Fig. 6: The slicing framework 
the next slice candidates. We present one such strategy, counterexample guided slicing, in Section 4.2 .

\subsection{Removing Instructions based on Heuristics}

The brute forcing (BF) strategy generates all possible slice candidates. As their number is exponential w.r.t. the number of instructions in the original program, it is clear that this strategy does not scale for large programs. Nevertheless, this strategy has the benefit of generating the smallest possible slice with our framework. Brute forcing can be used as part of a divide and conquer strategy to slice parts of programs which are small enough. As an improvement, this strategy can start by generating the candidates in ascending order with respect to their size, i.e. the number of instructions that the candidate retains from the original program. Once a candidate is shown to be a valid slice, no further candidates need to be checked, as their size cannot be smaller than that of the found slice.

The single statement elimination (SSE) strategy successively replaces a single instruction of the original program with skip, and checks whether the obtained program is a valid slice. If this is the case, the strategy attempts to successively remove every other instruction as well. The strategy requires, in the worst case, quadratically many calls to the relational verifier, which occurs when in each iteration the last candidate is shown to be a valid slice. Although this approach scales better than $\mathrm{BF}$, it finds only slices in which program instructions can be removed individually. Groups of instructions such as

assign $x(x+50)$

assign $x(x-50)$

where the removal of a single instruction results in an invalid slice candidate, but removing the entire group would result in a valid slice cannot be removed. The SSE strategy can be generalized to support the removal of groups of up to a given number of instructions.

\subsection{Counterexample Guided Slicing}

The counterexample guided slicing (CGS) strategy uses dynamic slicing to generate slice candidates. Dynamic slicing was first introduced in [23], and a survey on dynamic slicing approaches can be found in 24]. For the CGS strategy we adapted the dynamic slicing algorithm from [2, which is a syntactic approach based on on the Program Dependence Graph (PDG) [13. The PDG is a directed graph in which nodes represent program instructions, conditions, or input parameters, and edges represent possible dependencies between the nodes. An edge from node $n_{1}$ to node $n_{2}$ encodes that $n_{1}$ may depend on $n_{2}$. There are roughly two types of dependencies in the PDG. On one hand data dependencies arise when one node uses program variables which are defined in another node. Control dependencies, on the other hand, arise when the execution of a node depends on the other, control, node (e.g. an instruction may be executed only if the the condition of a conditional jump is true). Whether an edge exists between two 
nodes in the PDG is determined syntactically by analyzing the CFG. Because the CFG represents an over-approximation of the possible program executions, the PDG edges also represent an over-approximation of the real dependencies in the program. Using the PDG, a backward slice is computed by finding all nodes that are reachable from a node representing the criterion location. On the most basic level, the algorithm in [2, which receives the PDG and an execution trace as inputs, works by computing the subgraph of the PDG which contains only the nodes corresponding to those instructions which have been executed in the program trace. The dynamic slice is computed using this subgraph and further optimizations are possible, as it has to be valid only for a single input.

A PDG node can depend on multiple other nodes, but some of these dependencies are determined by the execution path of the program (e.g. a variable can be assigned on more than one branch, resulting in multiple dependencies for instructions that use that variable). Unlike static slicing, for dynamic slicing only one execution path is relevant - the one corresponding to the input for which the dynamic slice is computed. Thus, PDG edges representing dependencies that are relevant only for other inputs can be removed. A similar situation arises with loops: at different loop iterations, a node inside the loop body may have different dependencies. When performing dynamic slicing, the number of iterations done by a loop is known (assuming the program terminates for the input), and the PDG can be extended with nodes representing the body instructions at different iterations, which also leads to an increased precision of the dynamic slice. The extended PDG is called a dynamic dependence graph (DDG) in 2]. Based on the observation that the nodes inside the loop body can depend on only a finite number of other nodes, a new node is added to the PDG just for those iterations in which the corresponding instruction has different dependencies than in all previous iterations. This optimizations give rise to the reduced dynamic dependence graph (RDDG). Thus, by ignoring dependencies caused by other inputs than the one for which the dynamic slice is computed, additional instructions can be removed than in the case of static slicing. To ensure compatibility with the slicing property from Definition 4 , we adapt this algorithm to support criterion locations other than the end of the program. For this, when computing the dynamic slice with the RDDG we do not mark the return statement, as is done in 2, but rather all nodes that correspond to the criterion location. If the criterion location is inside a loop, then multiple nodes are marked.

The adapted RDDG dynamic slicing algorithm is purely syntactical and thus scales much better than a semantic approach. Thus we can use it as part of the candidate generation strategy, as relational verification of slice candidates remains the bottleneck of our framework. A property of the dynamic slices is the fact that the union of multiple dynamic slices can be computed.

Theorem 3. Let $P$ be a program and $s_{1}$ and $s_{2}$ be two initial states for which the respective dynamic slices $P_{d 1}$ and $P_{d 2}$ have been computed. The union of the dynamic slices $P_{d 1} \cup P_{d 2}$, that contains all instructions from $P$ which appear in $P_{d 1}$ or in $P_{d 2}$ is a dynamic slice for both initial states $s_{1}$ and $s_{2}$. 
Proof. The correctness of the dynamic slicing algorithm implies that in a dynamic slice for the initial state $s_{1}\left(s_{2}\right)$ only those instructions that do not influence termination and the values of the criterion variables are replaced with skip. If any of the replaced instructions are brought back, then the obtained program is still a valid dynamic slice for $s_{1}\left(s_{2}\right)$.

Using this property we can present the CGS strategy, shown in Algorithm 1 . It starts with an the initialization of the slice candidate $P_{s}$ with a program $\Phi$, in which all instructions have been replaced with skip, of an arbitrary initial state $s$, e.g. one in which all variables are set to 0 and of the variable $b$ which will be set to true when a valid slice will be found. The strategy uses the initial state $s$ with the criterion $\left(i_{C}, V_{C}\right)$ to compute a dynamic slice $P_{d}$. The instructions from $P_{d}$ are then added to the slice candidate $P_{s}$ which is checked for validity by the relational verifier. If $P_{s}$ is a valid slice candidate, the variable $b$ is set to true and the strategy returns $P_{s}$. Otherwise, the relational verifier delivers a counterexample, which is used as the initial state $s$ in the next iteration. Both the dynamic slicer and relational verifier may timeout, in which case the strategy returns the original program $P$.

Theorem 4. Let $P$ be a program and $P_{d}$ be a dynamic slice for all initial states $s \in S_{d}$, and $s_{c e}$ be the counterexample obtained when checking whether $P_{d}$ is a valid slice of $P$. Then the following holds:

1. $s_{c e} \notin S_{d}$.

2. The dynamic slice $P_{c e}$ for the initial state $s_{c e}$ contains at least one instruction which is not in $P_{d}$.

Proof. Both properties follow from the correctness of the relational verifier and of the dynamic slicer. (1) If $s_{c e} \in S_{d}$ then the relational verifier delivered a spurious counterexample or the dynamic slicer delivered an invalid dynamic slice. (2) If $P_{c e}$ contains no additional instruction compared to $P_{d}$, then $P_{d} \cup P_{c e}=P_{d}$ which means that $P_{d}$ is a dynamic slice for $s_{c e}$. This implies that the relational verifier delivered a spurious counterexample.

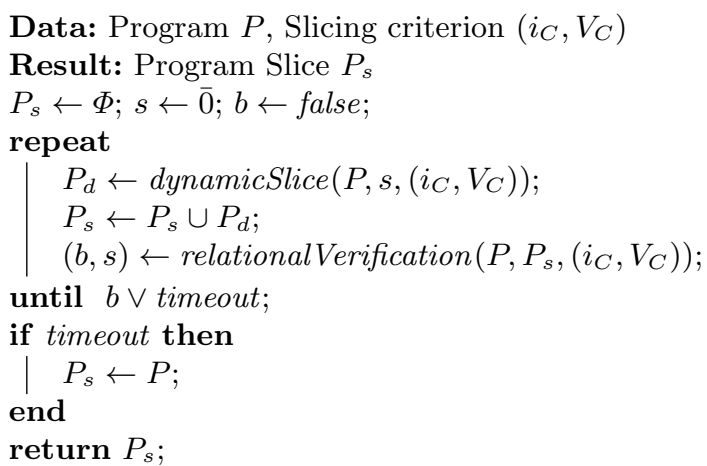

Algorithm 1: The CGS Strategy 
Theorem 4 guarantees that the CGS strategy adds at least one instruction back after each iteration (if no timeout occurred). Thus, the number of calls of the relational verifier is linear in the number of program instructions. The CGS strategy has the least number of calls to the relational verifier compared with the other strategies presented in this paper. Nevertheless, it comes with some disadvantages. First, the program needs to be executed at each iteration, which - depending on the analyzed program - can cause performance issues and for some inputs the program may not even terminate. Second, the CGS strategy is vulnerable to timeouts of the relational verifier, which are inevitable, as the program-equivalence problem is undecidable. If a timeout occurs, then the strategy fails entirely and must return the original program as the slice candidate, while the BF and SSE strategies could continue their search for a valid slice candidate. Third, the precision of CGS depends on the precision of the dynamic slicing approach used in the candidate generation. Even though dynamic slicing approaches which work on a syntactic level only can remove more statements than static slicing approaches, the dynamic slices they compute are still overapproximations, as they do not consider the semantics of the instructions.

\section{Discussion}

We start the discussion by reiterating the evaluation results of the implementation of the framework consisting of the tool SemSlice [5], as shown in Table 1. For the evaluation, we used a collection of small but intricate examples (e.g., the example of Fig. 1 1 or a routine in which the same value is first added and then subtracted), each focusing on a particular challenge for semantics-aware slicing was used. Some examples are taken from slicing literature [4/7/14/19]33. The second column indicates the source of each example, the third the number of LLVMIR statements in the program. For each slice candidate generation-method from Section 4 , the table lists the number of statements in the smallest slice found by SemSlice, the (wall) time needed by the tool, and the number of calls to the relational verifier. The experiments were conducted on a machine with an Intel Core I5-6600K CPU and 16GB RAM. The exponential BF approach works satisfactorily fast on functions with up to 20 statements, and while it requires more time than the other approaches it computes more precise slices. For examples with less than 10 statements the brute-force approach takes less than one second. The other two approaches achieved slices of similar precision (to each other) and required less than one second for most examples.

Our slicing approach works on an intermediate representation language. This is beneficial for the implementation of the approach, as it does not need to handle all features of a modern high level programming language. However, one of the uses for program slicing is to help the user debug and comprehend a program written a high level language. It is possible to perform relational verification of such programs, the early version of LLRÊVE was in fact working on a simple while language in [12, LLVM-IR was later chosen 21] to increase the practicability of LLRÊVE. We believe the current framework can be adapted 
Table 1: Evaluation

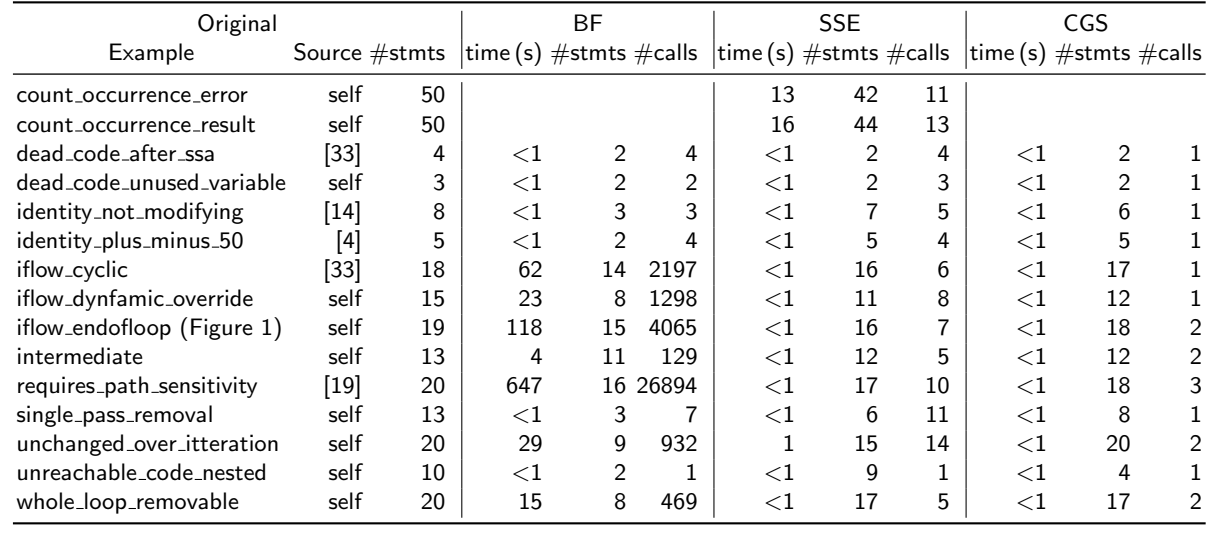

for slicing high level languages by either (1) attempting to translate back the IR slice to the high level language, or (2) by defining the slicing candidate in the high level language and then translating both the original program and the slice candidate into the IR and then using the extended relational verifier. For the first option we expect that only an over-approximation of the IR slice can be obtained by translating it back into the high level language, similar to what was done in [17. As for the second solution, the CFGs of the original program and slice candidate in the IR may be so different that our approach would no longer be able to automatically find matching synchronization points. A solution to this would be to automatically annotate the original program and its slice candidate in the high level language, thus marking the synchronization points and using this marks in the IR translation.

The IR language that we used to present our approach is not inter-procedural. While we could consider all programs as having been inlined beforehand, recursive procedures would not be supported. The relational verifier supports dealing with function calls using mutual function summaries 21] which abstract two matching function calls using coupling predicates. In general it is difficult to find matching function calls, but for checking the validity of slice candidates this can be done automatically, similar to finding matching synchronization points. Thus, our approach can be extended to support recursive functions; however the function calls themselves may not be removed, otherwise the mutual function summaries cannot be used.

The completeness of our approach, i.e. whether a valid slice is deemed as such, is limited by two factors. First, the relational verifier is required to automatically infer the coupling predicates needed to verify the validity of a slice candidate. In practice the relational verifier works well when the needed coupling predicates are limited to linear arithmetics 22. The second factor limiting completeness is the requirement that the original program and the slice candidate must run in lockstep. This is needed to ensure the mutual termination and that the criterion 
location is executed the same number of times. Thus, whereas we can remove instructions from inside a loop, we are not able to remove the loop itself (in our case the conditional jump instruction), even if it is empty - i.e. it loops over skip instructions. A possible solution to this is to check termination through other means and then remove empty loops that are guaranteed to terminate.

\section{Related Work}

Static slicing is an active area of research and many approaches have been developed. We present those that are most similar to our work.

Assertion based slicing [4] is also a slicing approach that takes the semantics of the program into consideration. Program methods must be specified with a contract, which also represents the slicing criterion, i.e. statements are removed that the reduced program still fulfills the contract. This approach combines preand postcondition-based slicing. Postcondition-based slicing works by computing the weakest precondition before every program location. If the weakest precondition at a location $i$ implies the weakest precondition at a location $j$ with $i<j$, then the instructions between the locations $i$ and $j$ may be removed, as they do not contribute to the truth value of the postcondition. Precondition-based slicing works similarly by using a strongest postcondition calculus. Unlike in our approach, loop invariants are required and only groups of instructions that are at consecutive program locations can be removed. This approach improves and combines older approaches [9]8, an implementation also exists [10].

The approach in 25] also uses a method's contract as the slicing criterion. Using a proof for a bounded number of loop iterations and type instances, it finds the parts of the program which were not needed for the bounded proof and constructs a slice candidate. However, the program parts that are deemed irrelevant are not removed, but replaced with an abstraction. Thus, the slice candidate over-approximates the behavior of the original program. If the contract is shown to be valid for the slice candidate, then it is also valid for the original program. A counterexample to the validity of the contract for the slice candidate can be used to generate a more concrete candidate, making this approach similar to the CGS strategy. For proving the contract of the slice candidate, loop invariants and user interaction are needed.

Path sensitive backward slicing [19] is another slicing approach that takes the program's semantics into consideration. The main idea is to symbolically execute the program and check the satisfiability of the path condition of every execution path. Only the satisfiable paths are used for computing the slice. The path explosion is mitigated by reusing the results of already performed satisfiability checks. The approach handles loops by using abstract interpretation to generate loop invariants, which can lead to an over-approximated description of the loop behavior. Thus, while the approach offers an increased precision when compared to syntactic approaches, it is not able slice the program in Fig. 1a. An implementation of this approach is available in the tool Tracer [20]. The idea of discarding dependencies that can only occur on infeasible program paths has 
also been explored in other works e.g. 3017. For these approaches, a compromise between the precision and scalability had to be found.

Abstract program slicing [15] is an approach which makes use of the program's semantics, however a different slicing criterion is used. Instead of preserving those instructions that affect the exact values of the criterion variables at the criterion location, this approach preserves the statements that affect a property of the criterion variable. The properties pursued in this approach are whether the variables belong to a given abstract domain, e.g. the positive integers. Using abstract interpretation, for some operations the abstract domain of the output is known - provided the abstract domains of the inputs are also known. Thus some dependencies modeled in the PDG can be removed. This approach can generate slices which are not valid according to Definition 4 .

The approach in 28. uses the formal semantics definitions of a language to automatically generate a slicer for programs written in that language. The approach to slicing works in two steps: first it analyzes the formal semantics definitions and computes for every instruction the parameters on which that instruction has side effects (data or control dependencies). This information is then used in the second step, in which for program and set of program variables, which constitutes the criterion, an over-approximated set of instructions that have a direct or indirect side effect on them is computed. This set constitutes the slice. The focus of this work lies, however, on the automatic generation of program slicers and not on the precision of the slices generated by them. This approach is implemented in the tool Chisel 3 .

Other, syntactic, slicing approaches have been surveyed in [35] and in [31, and a survey of dynamic slicing techniques can be found in [24].

\section{Conclusion and Future Work}

In this paper we extended a relational verification approach such that it can check whether a slice candidate is indeed a valid slice. Based on this, we built a framework for precise and automatic static slicing which consists of a candidate generation engine and the extended relational verifier. We presented three strategies to compute slice candidates, where the counterexample guided slicing is a more sophisticated approach. It uses the counterexample provided by the relational verifier to refine the slice candidate with a dynamic slicer.

As future work we plan to improve the precision of the slices by performing an additional analysis on empty loops to check whether they terminate. If this is the case, they can be removed without violating the slice property. Another research direction we wish to pursue is to improve the performance of the relational verifier by using PDGs to simplify the programs that need to be checked for equivalence, using the fact that two programs with isomorphic PDGs are equivalent, as shown in 18 . Furthermore, we will investigate how the results (e.g. coupling invariants) of the SMT solver employed by the relational verifier can be used when checking another slice candidate, constructed from the same original program. 


\section{References}

1. LLVM language reference manual. https://llvm.org/docs/LangRef.html. accessed: 2019-02-06

2. Agrawal, H., Horgan, J.R.: Dynamic program slicing. In: Proceedings of the ACM SIGPLAN 1990 Conference on Programming Language Design and Implementation. pp. 246-256. PLDI '90, ACM, New York, NY, USA (1990). https://doi.org/10.1145/93542.93576

3. Asăvoae, I.M., Asăvoae, M., Riesco, A.: Slicing from formal semantics: Chisela tool for generic program slicing. International Journal on Software Tools for Technology Transfer 20(6), 739-769 (Nov 2018). https://doi.org/10.1007/s10009018-0500-y

4. Barros, J.B., da Cruz, D., Henriques, P.R., Pinto, J.S.: Assertion-based slicing and slice graphs. Formal Aspects of Computing 24(2), 217-248 (Mar 2012). https://doi.org/10.1007/s00165-011-0196-1

5. Beckert, B., Bormer, T., Gocht, S., Herda, M., Lentzsch, D., Ulbrich, M.: Semslice: Exploiting relational verification for automatic program slicing. In: Polikarpova, N., Schneider, S. (eds.) Integrated Formal Methods. pp. 312-319. Springer International Publishing (2017). https://doi.org/10.1007/978-3-319-66845-1_20

6. Binkley, D., Harman, M.: A survey of empirical results on program slicing. Advances in Computers, vol. 62, pp. 105 - 178. Elsevier (2004). https://doi.org/10.1016/S0065-2458(03)62003-6

7. Canfora, G., Cimitile, A., Lucia, A.D.: Conditioned program slicing. Information \& Software Technology 40(11-12), 595-607 (1998). https://doi.org/10.1016/S09505849(98)00086-X

8. Chung, I.S., Lee, W.K., Yoon, G.S., Kwon, Y.R.: Program slicing based on specification. In: Proceedings of the 2001 ACM Symposium on Applied Computing. pp. 605-609. SAC '01, ACM, New York, NY, USA (2001). https://doi.org/10.1145/372202.372784

9. Comuzzi, J.J., Hart, J.M.: Program slicing using weakest preconditions. In: Gaudel, M.C., Woodcock, J. (eds.) FME'96: Industrial Benefit and Advances in Formal Methods. pp. 557-575. Springer, Berlin, Heidelberg (1996). https://doi.org/10.1007/3-540-60973-3_107

10. da Cruz, D., Henriques, P.R., Pinto, J.S.: Gamaslicer: An online laboratory for program verification and analysis. In: Proceedings of the Tenth Workshop on Language Descriptions, Tools and Applications. pp. 3:1-3:8. LDTA '10, ACM, New York, NY, USA (2010). https://doi.org/10.1145/1868281.1868284

11. De Angelis, E., Fioravanti, F., Pettorossi, A., Proietti, M.: Relational verification through Horn clause transformation. In: Rival, X. (ed.) Static Analysis. pp. 147169. Springer, Berlin, Heidelberg (2016). https://doi.org/10.1007/978-3-662-534137_8

12. Felsing, D., Grebing, S., Klebanov, V., Rümmer, P., Ulbrich, M.: Automating regression verification. In: Proceedings of the 29th ACM/IEEE International Conference on Automated Software Engineering. pp. 349-360. ASE '14, ACM (2014). https://doi.org/10.1145/2642937.2642987

13. Ferrante, J., Ottenstein, K.J., Warren, J.D.: The program dependence graph and its use in optimization. ACM Trans. Program. Lang. Syst. 9(3), 319-349 (Jul 1987). https://doi.org/10.1145/24039.24041

14. Field, J., Ramalingam, G., Tip, F.: Parametric program slicing. In: Proceedings of the 22Nd ACM SIGPLAN-SIGACT Symposium on Principles of Program- 
ming Languages. pp. 379-392. POPL '95, ACM, New York, NY, USA (1995). https://doi.org/10.1145/199448.199534

15. Halder, R., Cortesi, A.: Abstract program slicing on dependence condition graphs. Science of Computer Programming 78(9), 1240 - 1263 (2013). https://doi.org/10.1016/j.scico.2012.05.007

16. Hammer, C., Snelting, G.: Flow-sensitive, context-sensitive, and object-sensitive information flow control based on program dependence graphs. International Journal of Information Security 8(6), 399-422 (Dec 2009). https://doi.org/10.1007/s10207009-0086-1

17. Herda, M., Tyszberowicz, S., Beckert, B.: Using dependence graphs to assist verification and testing of information-flow properties. In: Dubois, C., Wolff, B. (eds.) Tests and Proofs. pp. 83-102. Springer International Publishing (2018). https://doi.org/10.1007/978-3-319-92994-1_5

18. Horwitz, S., Prins, J., Reps, T.: On the adequacy of program dependence graphs for representing programs. In: Proceedings of the 15th ACM SIGPLAN-SIGACT Symposium on Principles of Programming Languages. pp. 146-157. POPL '88, ACM, New York, NY, USA (1988). https://doi.org/10.1145/73560.73573

19. Jaffar, J., Murali, V., Navas, J.A., Santosa, A.E.: Path-sensitive backward slicing. In: Miné, A., Schmidt, D. (eds.) Static Analysis. pp. 231-247. Springer (2012). https://doi.org/10.1007/978-3-642-33125-1_17

20. Jaffar, J., Murali, V., Navas, J.A., Santosa, A.E.: Tracer: A symbolic execution tool for verification. In: Madhusudan, P., Seshia, S.A. (eds.) Computer Aided Verification. pp. 758-766. Springer, Berlin, Heidelberg (2012). https://doi.org/10.1007/978-3-642-31424-7_61

21. Kiefer, M., Klebanov, V., Ulbrich, M.: Relational program reasoning using compiler IR - combining static verification and dynamic analysis. Journal of Automated Reasoning 60(3), 337-363 (Sep 2017). https://doi.org/10.1007/s10817-017-9433-5

22. Klebanov, V., Rümmer, P., Ulbrich, M.: Automating regression verification of pointer programs by predicate abstraction. Formal Methods in System Design 52(3), 229-259 (Jun 2018). https://doi.org/10.1007/s10703-017-0293-8

23. Korel, B., Laski, J.W.: Dynamic program slicing. Inf. Process. Lett. 29(3), 155-163 (1988). https://doi.org/10.1016/0020-0190(88)90054-3

24. Korel, B., Rilling, J.: Dynamic program slicing methods. Information \& Software Technology 40(11-12), 647-659 (1998). https://doi.org/10.1016/S09505849(98)00089-5

25. Liu, T., Tyszberowicz, S., Herda, M., Beckert, B., Grahl, D., Taghdiri, M.: Computing specification-sensitive abstractions for program verification. In: Fränzle, M., Kapur, D., Zhan, N. (eds.) Dependable Software Engineering: Theories, Tools, and Applications. pp. 101-117. Springer International Publishing (2016). https://doi.org/10.1007/978-3-319-47677-3_7

26. Lucia, A.D.: Program slicing: methods and applications. In: Proceedings First IEEE International Workshop on Source Code Analysis and Manipulation. pp. 142-149 (Nov 2001). https://doi.org/10.1109/SCAM.2001.972675

27. de Moura, L., Bjørner, N.: Z3: An efficient smt solver. In: Ramakrishnan, C.R., Rehof, J. (eds.) Tools and Algorithms for the Construction and Analysis of Systems. pp. 337-340. Springer, Berlin, Heidelberg (2008). https://doi.org/10.1007/978-3540-78800-3_24

28. Riesco, A., Asăvoae, I.M., Asăvoae, M.: A generic program slicing technique based on language definitions. In: Martí-Oliet, N., Palomino, M. (eds.) Recent Trends in Algebraic Development Techniques. pp. 248-264. Springer, Berlin, Heidelberg (2013). https://doi.org/10.1007/978-3-642-37635-1_15 
29. Rümmer, P., Hojjat, H., Kuncak, V.: Disjunctive interpolants for Horn-clause verification. In: Sharygina, N., Veith, H. (eds.) Computer Aided Verification. pp. 347363. Springer, Berlin, Heidelberg (2013). https://doi.org/10.1007/978-3-642-397998_24

30. Snelting, G., Robschink, T., Krinke, J.: Efficient path conditions in dependence graphs for software safety analysis. ACM Trans. Softw. Eng. Methodol. 15(4), 410-457 (Oct 2006). https://doi.org/10.1145/1178625.1178628

31. Tip, F.: A survey of program slicing techniques. Tech. rep., Amsterdam, The Netherlands, The Netherlands (1994), https://www.franktip.org/pubs/ jpl1995.pdf

32. Verdoolaege, S., Janssens, G., Bruynooghe, M.: Equivalence checking of static affine programs using widening to handle recurrences. ACM Trans. Program. Lang. Syst. 34(3), 11:1-11:35 (Nov 2012). https://doi.org/10.1145/2362389.2362390

33. Ward, M.: Properties of slicing definitions. In: 2009 Ninth IEEE International Working Conference on Source Code Analysis and Manipulation. pp. 23-32 (Sep 2009). https://doi.org/10.1109/SCAM.2009.12

34. Weiser, M.: Program slicing. In: Proceedings of the 5th International Conference on Software Engineering. pp. 439-449. ICSE '81, IEEE Press, Piscataway, NJ, USA (1981), http://dl.acm.org/citation.cfm?id=800078.802557

35. Xu, B., Qian, J., Zhang, X., Wu, Z., Chen, L.: A brief survey of program slicing. SIGSOFT Softw. Eng. Notes 30(2), 1-36 (Mar 2005). https://doi.org/10.1145/1050849.1050865 